\title{
A New Method for Generating a Zero Mean Self-Balanced Orthogonal Chaotic Spread Spectrum Codes
}

\author{
Hany A. A. Mansour and Yongqing Fu \\ College of Information \& Communication Engineering, \\ Harbin Engineering University, Harbin 150001, China \\ hnhn118799@gmail.com, fuyongqing@hrbeu.edu.cn
}

\begin{abstract}
Spread spectrum technique becomes one of the most important êmmunication techniques in the recent decades. It provides high security, in addition to its resistance to the various interference and fading types. One of the most important parameter in the spreading process is the spreading code used in the spreading process, especially in the direct sequence spread spectrum. Recently, many researches focused on the chaotic codes, due to its attractive security properties, in addition to its availability to generate a huge number of spreading codes. This property is very useful in the multi-access applications. Howeyer, as the number of the MAI is increased, the performance of the chaoticcodes is degraded. This paper presents a new method to generate a set of orthogonal self-balanced chaotic codes. The results show that the presented method generates a set of chaotic codes with correlation properties much better than the traditional chaotic code. The results show also that the BER performance of the presented orthogonal chaotic codes is superior and outperforming the BER performance of the traditional chaotic codes in addition to the PN sequences.
\end{abstract}

Keywords: Spread Spectrum, Chaotic Code, Direct Sequence Spread Spectrum, and Gold Sequence..

\section{Introduction}

Since the Federal Communication Commission (FCC) has been established, its main basis in managing the spectrum allocations was depending on the request-by-request basis. As the communications applications are hugely increased, it has realized that it had no more spectra to allocate [1]. One of the available solutions of this problem is to share the same spectrum by multiple applications through the spread spectrum. The spread spectrum simply can be defined as the transmission way in which the signal occupies a bandwidth much wider than its original band width. This wider bandwidth is obtained by using certain spreading code completely independent on the message signal, and has much higher bit rate than the original signal. This spreading code is synchronized in the receiver to retrieve the original signal by means of the despreading process [2]. Generally, the spread spectrum techniques can be classified according to the way in which the information data is injected by the spreading code. The main types of spread spectrum are the direct sequence spread spectrum (DSSS), frequency hopping spread spectrum (FHSS), time hopping spread spectrum (THSS), chirp spread spectrum (CSS) and hybrid spread spectrum [3].

In direct-sequence spread spectrum (DS-SS) systems, the information signal is modulated by the spreading code prior the transmission, resulting in a wideband signal resistant to 
narrowband jamming, multipath, interception, in addition to providing the multiple-access capability. Typically, there are many codes types that can be used for the spreading process. The pseudo-noise $(\mathrm{PN})$ sequences, which are produced by the linear feedback shift register, are the most popular codes used in the multiusers application. Although PN code has attractive properties, however it has also a lot of drawbacks such as the limitation of the number of such sequences, in addition to its periodicity feature, which make the intercepted signal is predictable and is reconstructed by linear regression which leads to security limitations.

One of the alternative solutions is the long codes and chaotic code signals. Chaotic codes have a lot of attractive properties over the conventional PN sequences, especially from the security point of view. It is non-periodic, wide band, and more difficult to predict and to reconstruct. Moreover, Chaos is a deterministic, random-like process found in non-linear dynamical system, which is non-converging and bounded [4]. It has also a very sensitive dependence upon its initial condition and parameters, which gives the availabjity to generate an infinite number of spreading codes [5]. These properties make chaotic signals more difficult to intercept, and more secured to decode the information spreaded upon them [6]. In recent decades, many researchers have studied and discussed the properties of the chaotic codes from different point of views. The correlation properties is one of the most important point of views that has been studied and discussed in detail specially the cross-correlation properties, since it has a direct effect on the performance of the multi-access systems. These studies lead to create many types of maps that are used to generate the chaotic sequences, such as logistic map, tent map, Gauss iterated map, Gingerbread man map, and-Henon map [7]. Since not all of the chaotic maps can outperform the PN performance, it was necessary to study and create new methods to improve the correlation properties of the chaotic codes, and hence improve its performances.

This paper introduces a new method to generate a zero mean self-balanced orthogonal (ZMSBO) chaotic sequences using any generating map. The main idea depends on generating one zero mean self-balanced (ZMSB) chaotic sequence as a basic code, and then generates the other orthogonal sequences oy using a created orthogonal function. The method constructed mainly from four different stages, (i) the first stage is to generate the basic real value chaotic sequence from any available chaotie map, (ii) the second stage is to map the real chaotic values of the basic chaotie sequence into zeromean (ZM) binary sequences, (iii) the third stage is to generate the self balanced (SB) basic chaotic sequence from the ZM binary sequence [8], and finally (iv) the fourth stage is to generate the zero mean self-balanced orthogonal (ZMSBO) chaotic sequences set from the basic chaotic sequence. Due to the presence of a large number of chaotic maps, the logistio map which is the most popular chaotic map is considered in the simulation. The auto-correlation and the cross-correlation properties of the chaotic sequence generated from the presented method, traditional chaotic code, and the gold code are compared under the same conditions. Also, the BER performances of the presented chaotic code, traditiona chaotic code, and the gold code are compared in case of the flat fading channel. The results show that the chaotic code generated from the proposed method has correlation properties much better and superior performance over the traditional chaotic code, and the gold code.

The paper is organized as follows, Section 2 discusses the detailed steps of generating the ZMSBO chaotic sequences, the results and the different comparisons of the correlation properties and BER performances are illustrated in Section 3, and finally the conclusion is presented in Section 4. 


\section{Generating the ZMSBO Chaotic Sequence}

\subsection{Generate the Basic Real Value Chaotic Sequence}

As mentioned in the previous section, the logistic map represented by (1) is considered with the proposed method:

$$
x_{i+1}=R x_{i}\left(1-x_{i}\right), \quad x_{i} \in(0,1)
$$

Where, $x_{i+1}$ is the new value generated from the old value $x_{i}, R$ is the bifurcation parameter. As well known, the initial value $x_{0}$ affects on the rest of the generated values, which gives to the chaotic codes its main advantage of generating the huge number of the spreading sequences.

\subsection{Generate the Basic ZM Binary Chaotic Sequence}

In this stage, the real values of the basic chaotic sequence are mapped to the binary values. Actually, there various methods can be used to map the real values of the chaotic code into the binary values such as chaotic bit sequence, chaotic threshola sequence, and the chaotic mean sequence [9]. In this paper, the third method, which can be represented by (2), will be used.

$$
X_{1}=\operatorname{sign}(g\{x(t)-\operatorname{mean}[x(t)]\})
$$

This method depends on shifting the real value for the basicenaotic code by its mean value and generates a new basic sequence $g(x)$ has azero mean The binary values will then obtained by taking the sign function of the new zero mean sequence $g(x)$.

\subsection{Generate the Basic SBZM Chatic Sequence}

In this stage, the self-balancing process is performed in which the process is explained in detail in [8]. Its idea is briefly depends on four main steps which are, inversion, all upside down, radix-S block upside down, and finally the shift combination. As indicating in Figure 1, after the basic real valued sequence is converted to the basic binary sequence with length $N$, the inversion process is performed bultiplying -1 to the $\mathrm{ZM}$ basic sequence $X_{1}$, which can be represented mathènatically by (3) as:

$$
f\left(x_{i}\right)=-x_{i}, \quad M \leq i \leq M+N
$$

Where $M$ is the tarting point, and $2 N$ is denotes the spreading code length. The object of the starting point $M$ is to avoid the similarity that can be happened between the first values of the generated chaotic codes due to the slight change of the initial condition. In the presented system, only the basic chaotic sequence is generated, and hence $M$ can be set to zero. The inverted sequence is converted to the all up-side down sequence by arranging the original sequence in the opposite direction as cleared in (4):

$$
y_{i}=-x_{2 M+N-1-i}, \quad M \leq i \leq M+N
$$

The radix-S operation is proceeding on the upside down sequence $y_{i}$ with the segment length of $N$ as illustrated in Figure 2. In this step, the sequence is divided into $N / S$ short segments with length of $S$ for each. Then, each short segment is arranged in the opposite direction by itself. The final form is obtained by make the combination between all the $N / S$ inter-upsidedown short segments as represented in (5). 


$$
\begin{gathered}
z_{i}=y_{2 M+(2 j-1) S-1-i}=-x_{N-(2 j-1) S+1} \\
M \leq i \leq M+N, j=1,2, \ldots, N / S
\end{gathered}
$$

Where $j$ represents the short segments index number, which is a positive integer doesn't exceeds $N / S$. Finally, the combination process is performed between the radix-S block upside down sequence $z_{i}$ and the original sequence $x_{i}$, which are both has the length of $N$. The final zero mean self-balanced sequence $C_{1}$ is produced according to (6) as:

$$
C_{k}=\left\{\begin{array}{lc}
x \frac{k}{2}+M & k \in \text { even } \\
z \frac{k-1}{2}+M & k \in \text { odd }
\end{array}, k=0,1,2, \ldots, 2 N-1\right\}
$$

Where $k$ is the index number of the final ZMSB chaotic spreading code with length $2 N$.

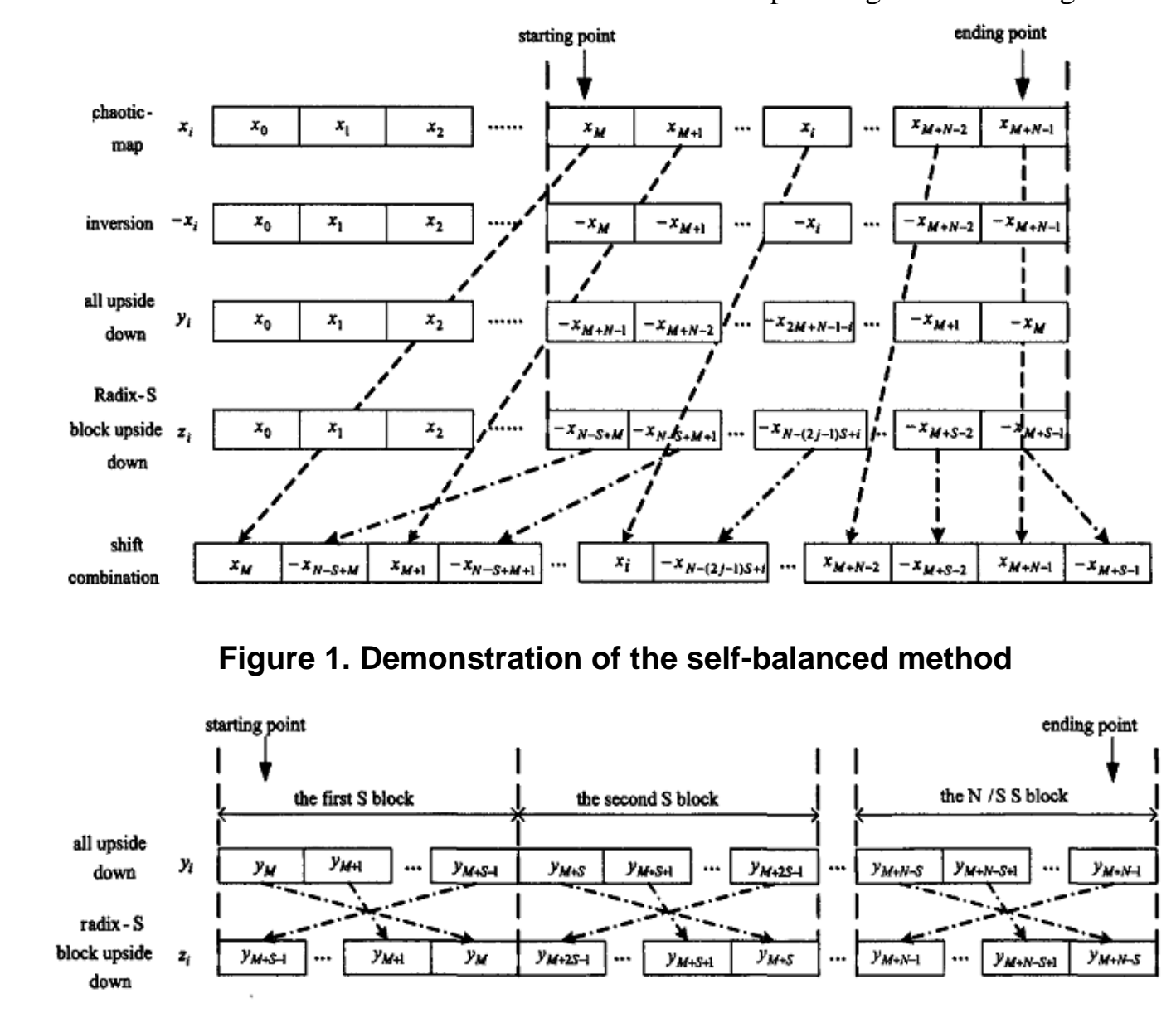

Figure 2. the illustration of the of the radix-S block upside down

\subsection{Generating the Orthogonal ZMSB Chaotic Code.}

The main idea in generating the orthogonal chaotic set is to multiply the basic ZMSB chaotic code with a certain orthogonal function. This function is constructed from the orthogonal matrix $F$ which has a dimension of $K \times 2 N$, where $K$ is the number of codes required to be generated according to the number of users, $2 N$ is the code length as indicated before. Each 
row in the orthogonal matrix $F$ is generated from 1 , and -1 such that sum of each row equal zero (i.e. the number of 1's equal to the number -1 's with different orders). The orthogonal codes are generated as the result of the dot product multiplication between the basic ZMSB code and the corresponding row in the orthogonal matrix $F$.

For the comparison purposes with the PN sequences, it is assumed that the chaotic code length is subjected to the binary system. Consequently, the matrix $F$ is created as follows:-

a. Generate a vector $v$ of length $2 N$ contains the values 1 and -1 alternately.

b. Create $n$ levels, where $n=\log _{2}(2 N)$ or $2^{n}=2 N$, such that the $n t h$ level representing the half division of the vector $v$ into 2 similar segments with length $N$ for each. The $(n-1) t h$ contains 4 similar segments with length $N / 2$ for each; representing the half division of each segment in the first level, and so on until reach to the first level which contains $N$ segments with length 1 for each.

c. The first row in the matrix $F$ constructed from the $2 N$ elements of the first lever with exchanging the first two segments. The second row is the same level with exchanging the second two segments, and so on until the Nth row.

d. To form the $(N+1)$ th row, the first two segments in the secônd level are exchanged, and so on until the $(N+N / 2)$ th row. Generally, the row number $\frac{N}{2^{(l-1)}}(l-1)+i$ will be constructed by exchanging the two segments $s_{2}=-s_{2 i-1}$ in the level $l$ where $=$ $1,2, \ldots, N / 2^{(l-1)}$.

e. By continuing this process until the $n$th level, a 2N/ 1 row will be performed corresponding to $2 N-1$ user. This $2 N$ code length is corresponding to one symbol duration. For $R$ symbols the available number of ows will be $2 R N-1$, which leads to provides $2 R N-1$ users.

In general, for code length is subjected to the binary system, the total number of the available chaotic codes will be calculated according to (75)

$$
R \sum_{i=1}^{n} 2^{i} Q^{1,} \quad n=\log _{2}(2 N)
$$

Figure 3 illustrates the process of creating the orthogonal matrix $F$. The main drawback of this method is the limitation of the number of spreading codes, however it is clear that the number of the generated codes (and hence the number of available users) are proportional to the number of the message symbels.

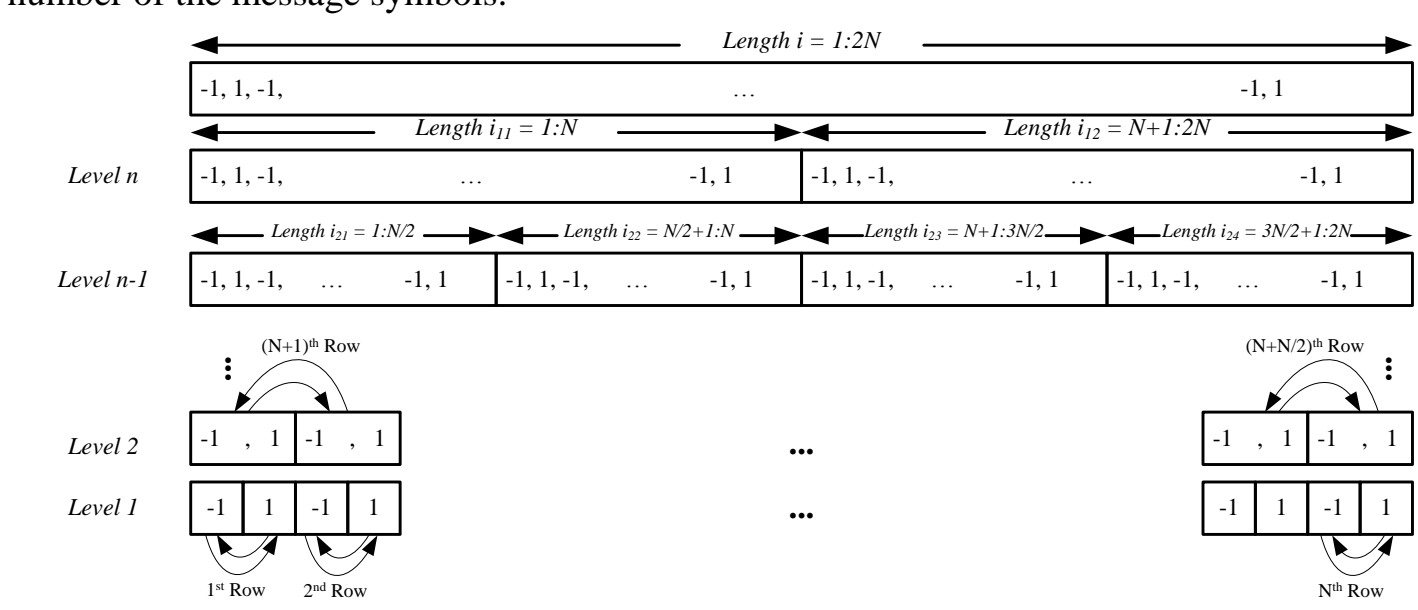

Figure 3. Illustration of the Orthogonal Matrix Construction 


\section{Simulation results}

In this section a comparison between the orthogonal chaotic code, traditional chaotic code and gold code is performed from many points of views. Regarding to the correlation properties, Figure 4, and 5 discusses the autocorrelation function (ACF) and the cross correlation (CCF) for the logistic traditional chaotic code, logistic orthogonal chaotic code, and the gold code for lengths 127 and 255.

In Figure 4, the results for the code length 127 show that, although the orthogonal chaotic code has some peaks with large amplitudes over \pm 20 , however generally its CCF is much better than that of the traditional chaotic and the gold code, since its majority values are within \pm 10 , its zero mean, in addition to its noise random like. For the ACF, it is found that the orthogonal code has better ACF than that of the traditional chaotic code, since its side loops variation has zero mean, in addition to it has lower values.

Figure 5 shows the same results for the code length 255. It is clear that the CF of the orthogonal code has some peaks with amplitudes larger \pm 40 . However, its majority peaks are noise like has amplitude between \pm 20 with zero mean. This leads to be still better than that of the traditional chaotic and gold code.
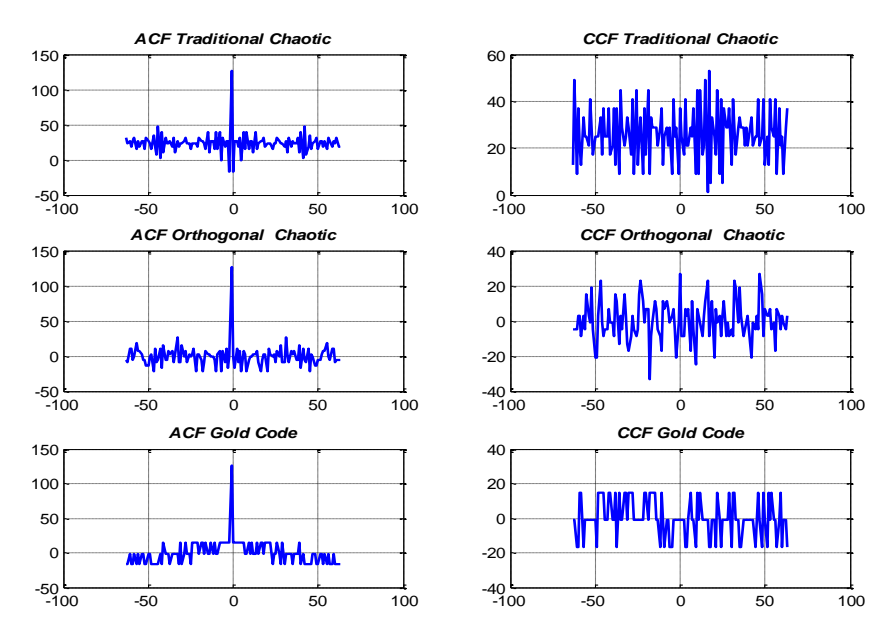

Figure 4. Comparisons of Correlation Properties for Length 127
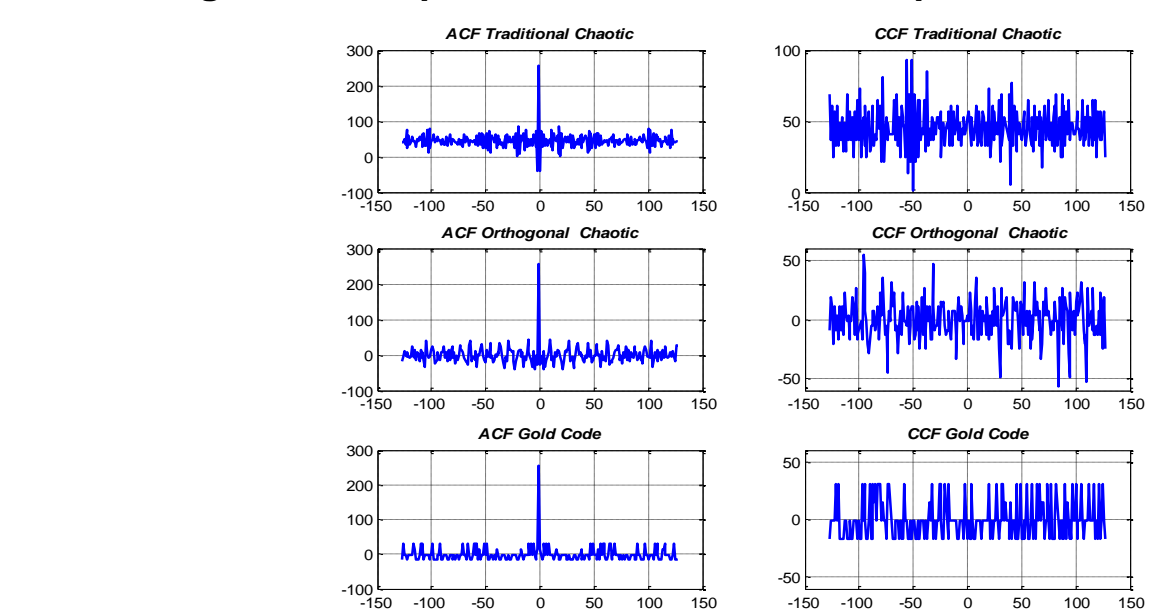

Figure 5. Comparisons of Correlation Properties for Length 255 
In Figures 6, the cross correlation values between the first users and the rest of the other users in the CDMA system is calculated and plotted for the traditional and orthogonal chaotic codes. The Figure shows that for code length 127 and in presence of 120 user, , the cross correlation between the desired user and any other user for the orthogonal code is zero, which mean that all the other codes is completely orthogonal on the desired user. Meanwhile in the case of the traditional chaotic code and under the same condition, the cross correlation values between the desired user and the other users are varying between 40 and -30 .

Figure 7 has the same comparison of Figure 7 but with code length 511 and in presence of 500 different users. The result shows also that all the interfering users are completely orthogonal to the desired user, as the cross correlation value between all the users and the desired one is zero. In the case of the traditional chaotic code, it's found that the cross correlation properties are varying between 80 and -70 . These two Figures give an indication that in case of the orthogonal chaotic code, all the interfering users are completely eliminated.

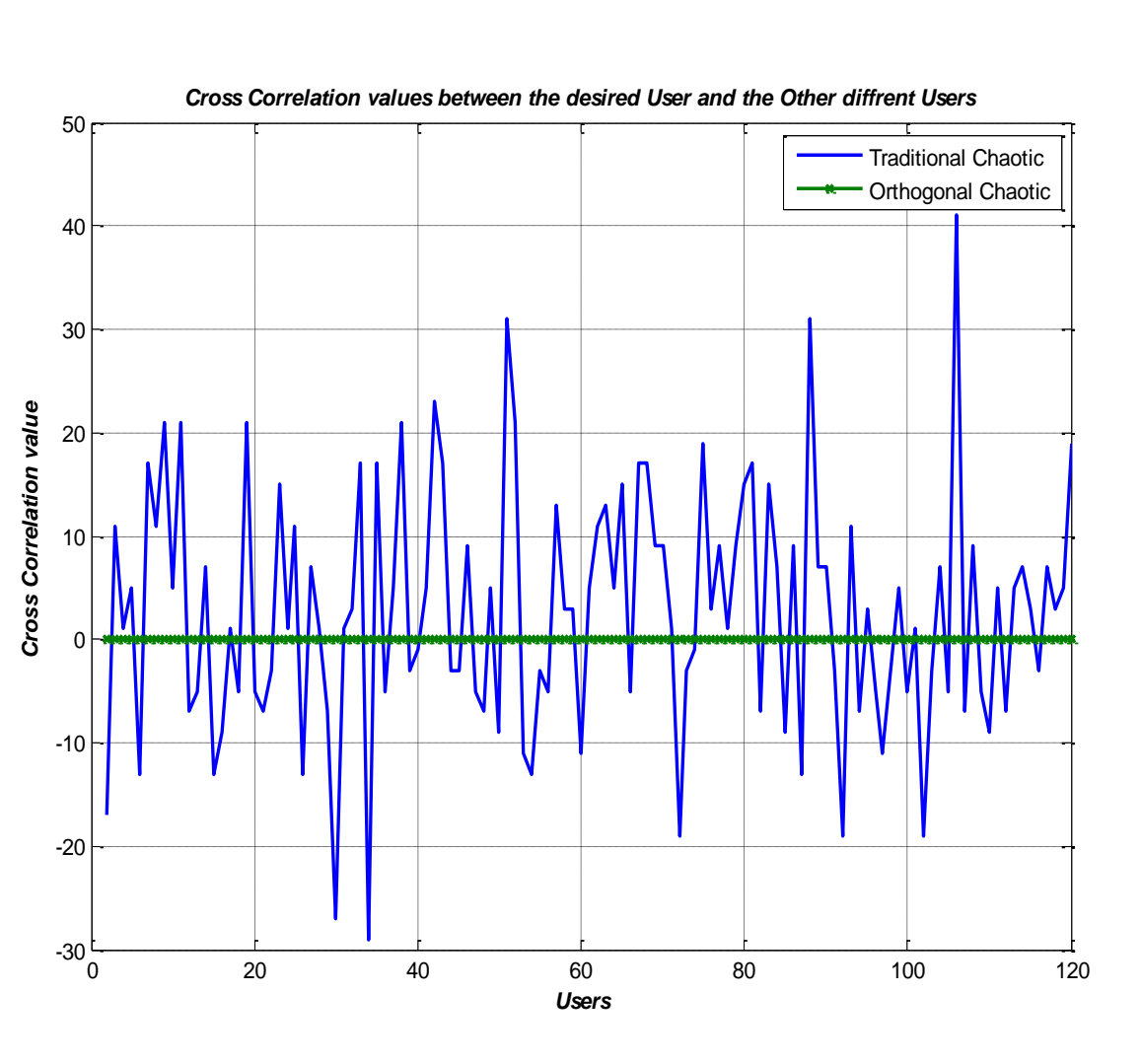

Figure 6. Comparisons of Correlation values between the different users (Length $B^{\circ}$ 


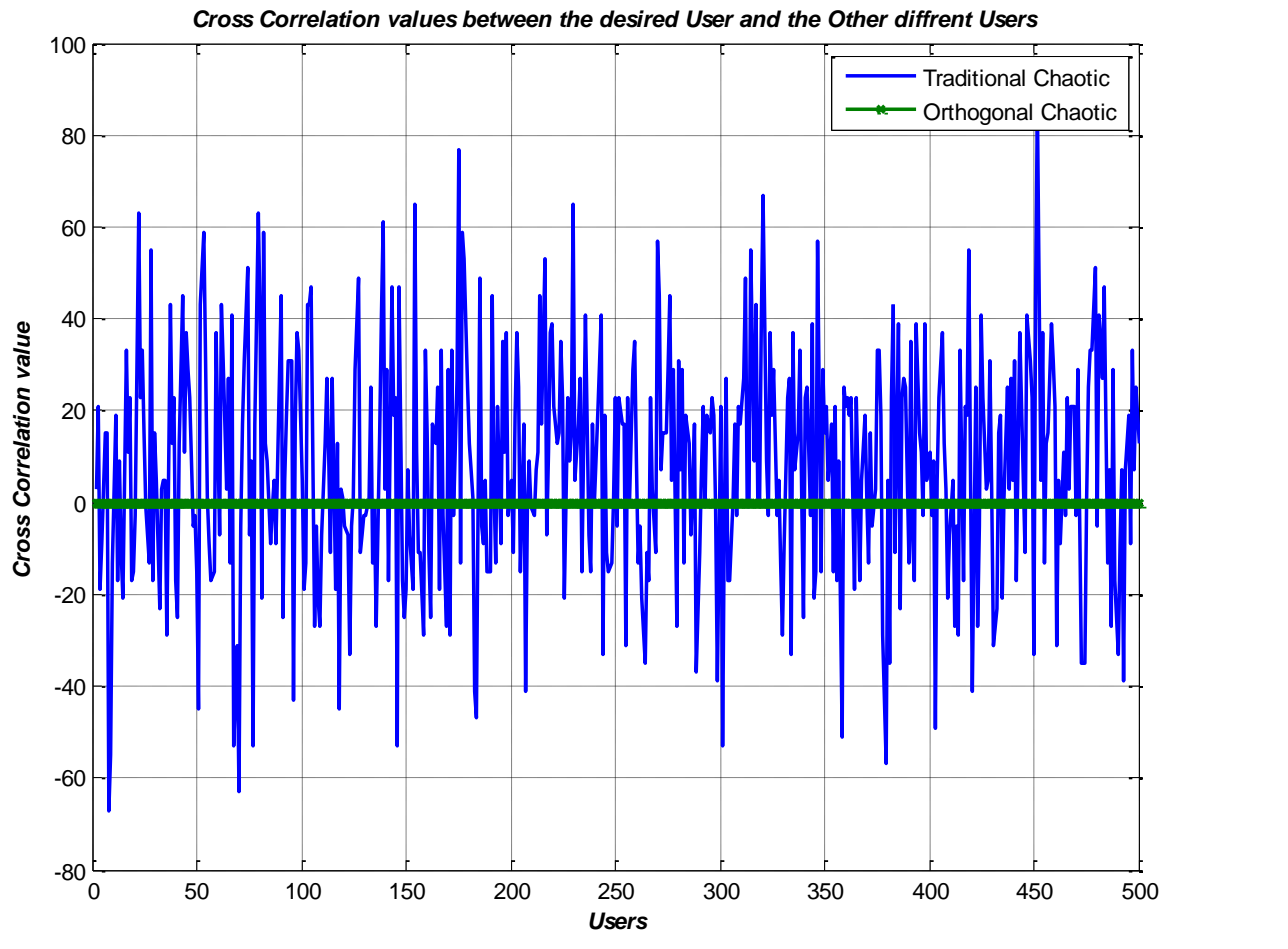

Figure 7. Comparisons of Correlation values between the different users (Length

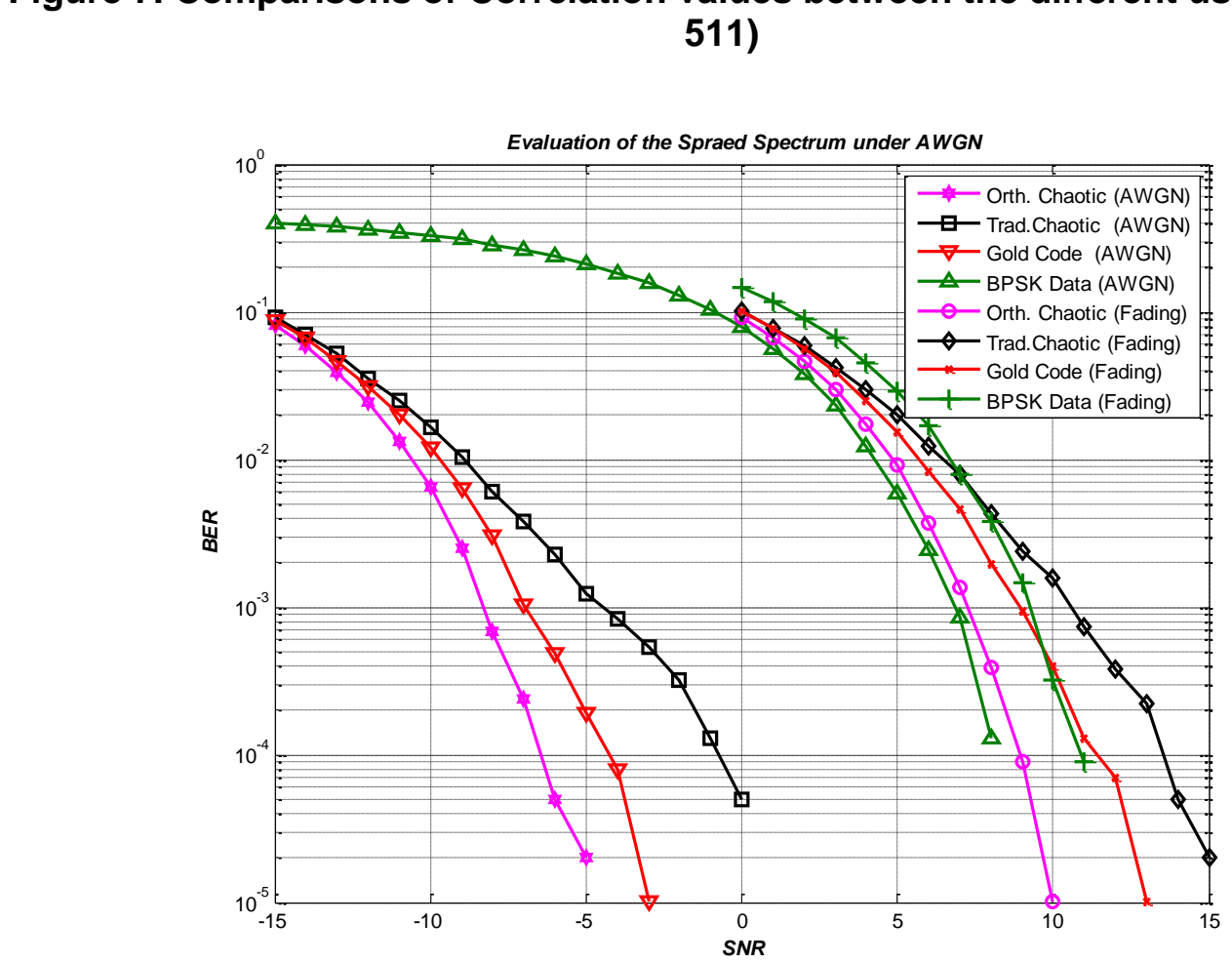

Figure 8. Performance comparisons under different channels 
Regarding to the bit error rate, Figure 8 shows the performance comparison between the traditional chaotic code, orthogonal chaotic code, and the gold code in addition to the traditional BPSK. The comparisons are performed in the AWGN channel and Relighy flat fading environment using BPSK DSSS communication system. The simulation parameters are set as baseband data rate $10^{4} \mathrm{bps}$, carrier frequency $2.4 \mathrm{GHz}$, and the spreading code length is chosen to be 31, in presence of 30 interfering user. The result shows that in case of the AWGN channel, the orthogonal chaotic code completely eliminates the MAI effect and presents a performance gain about $2 \mathrm{~dB}$ at BER $10^{-4}$ over the gold code, and $5 \mathrm{~dB}$ at BER $10^{-4}$ over the traditional chaotic code. Obviously, the spreading gain due to the spreading code length (31) is about $14.5 \mathrm{~dB}$ at BER $10^{-4}$ over the traditional single user BPSK. In the case of the Relighy flat fading, the performance is performed under sever fading conditions, in presence of 30 MAI. The plot shows that the performance of the orthogonal chaotic code is still much better than the performance of the traditional code and the gold code due to the improvemen of its cross correlation properties. It clear that the performance of the orthogonal code presents more than 2 $\mathrm{dB}$ gain at BER $10^{-4}$ over the gold code performance, and outperforms the traditional code by more than $4.5 \mathrm{~dB}$ gain at BER $10^{-4}$. It can be shown that the performance of the orthogonal code under the fading channel in presence of 30 MAI jo nearly the same of the single user BPSK performance under AWGN. This mean that the presented method provides a superior code performance since it counters the effect of the large MAI number in the fading conditions

\section{Conclusion}

The new contribution of this paper is a new method to generate an orthogonal zero mean self-balanced chaotic code set. The presented method can be applied to any chaotic map with any code length. A comparison is performed between the orthogonal code generated with the presented method, a traditional chaotic code, and a gold code from different point of views. The results shoes that the correlation properties and the BER performance of the presented method is much better than the other codes.

\section{References}

[1] R. Billa, "Analys of Direct Sequence and Chirp Spread Spectrum over Flat Fading Channel for Multiple Access", IJCEM vol. 15, issue 5 (2012).

[2] C. Turgay, "Fast and Efficiency Method for Fire Detection Using Image Processing", ETRI Journal, vol. 32, (2010), pp.881-890.

[3] R. Nawkhare and A. Tripathi, "DS-SS Communication system using pseudo chaotic sequences generator", Proceedings of the international Conference on Communication Systems and Network technologies (2013).

[4] T. S. Rappaport "Wireless Communications: Principles and Practice", Prentice Hall PTR, NJ, (2009).

[5] H. Palubová, "The type of chaotic sequences for signal transmission", Proceedings of the 10th Scientific Conference of Young Researchers - FEI TU of Košice (2010).

[6] R. Vali, S Berber, and S. K. Nguang, "Accurate derivation of chaos-based acquisition performance in a fading channel”, JEEE Trans. Wireless Communication., vol. 11, no. 2, (2012) February, pp. 722-731.

[7] G. Kaddoum, D. Roviras, P. Charge and D. Fournier-Prunaret, "Performance of multi-user chaos-based DSCDMA System over multipath channel", IEEE Trans, (2009).

[8] S Kim, J. Bok and H. Ryu, "Performance Evaluation of DCSK System with Chaotic Maps", 978-1-4673-57425/13/\$31.00 IEEE (2013).

[9] A. Yong, M. Rongzeng, C. Xiaolin and Y. Yeupeng, "An Improved Method of Generating the Self-Balanced Chaotic Spread-Spectrum Code”, HIGH TECHNOLOGY LETTERS, vol. 17, no. 1, (2011) March, pp. 46-51.

[10] G. V. Reddy, "Performance Evaluation of Different DS-CDMA Receivers Using Chaotic Sequences", Master Thesis, (2007). 


\section{Author}

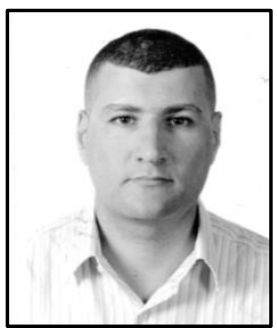

Hany Amin Mansour, he was born in Suez, Egypt on July 5, 1979. $\mathrm{He}$ received his B.S. (Excellent with honors) and M.S. in communication engineering from the Military Technical College (MTC), Cairo, Egypt in 2002, and 2009, respectively. He served as an assistant teacher in the MTC from 2004 to 2011. Since 2012, he has been pursuing the Ph.D. degree with the Information and Communication Department, Harbin Engineering University (HEU), Harbin, China. His research interests include the spread spectrum, chaotic codes, and adaptive filtering techniques.

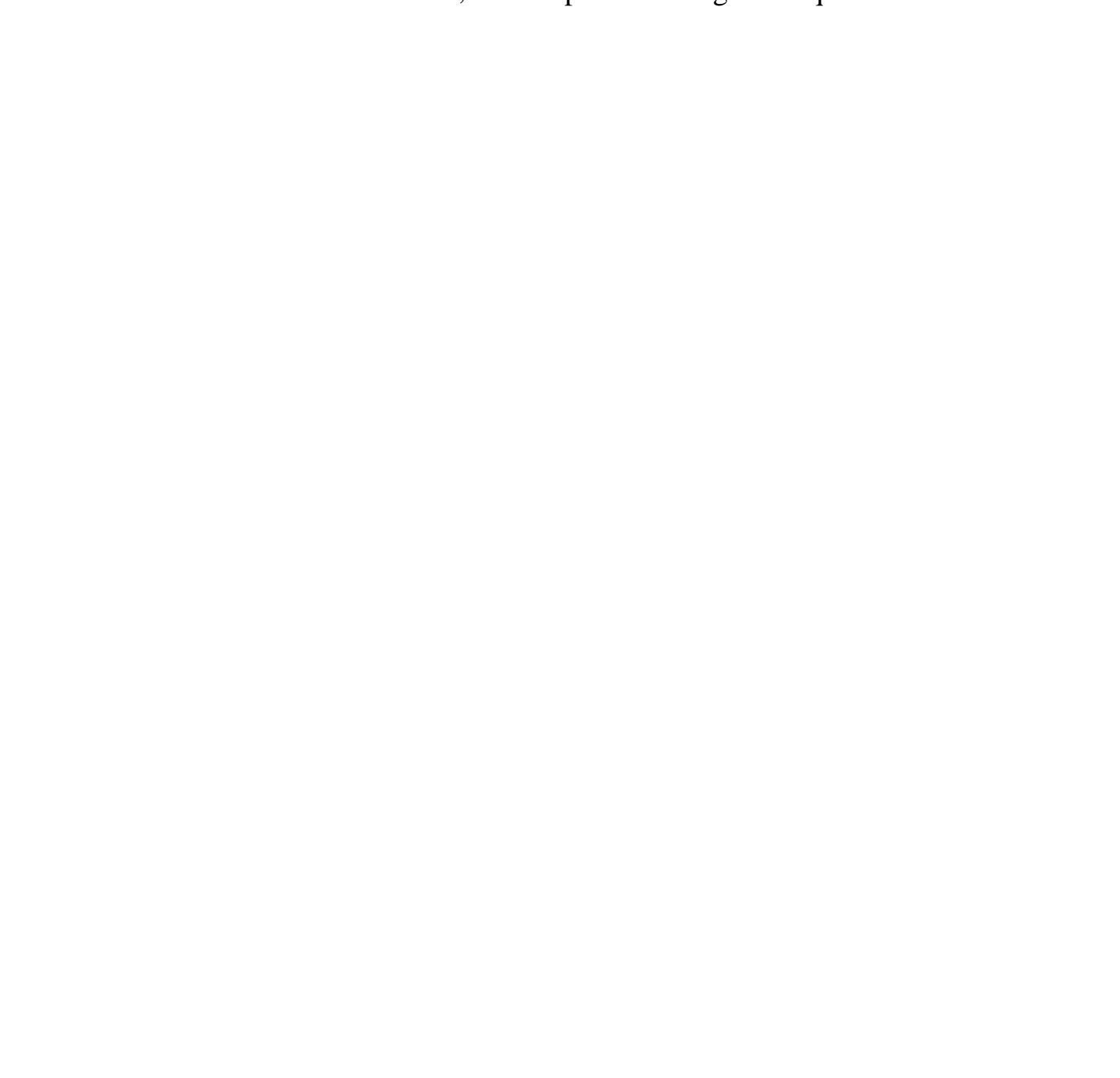

